\title{
Noninvasive control of the transport function of fluorescent coloured liposomal nanoparticles
}

\author{
O. Stelmashchuk ${ }^{1}$, E. Zherebtsov ${ }^{1,2}$, A. Zherebtsova ${ }^{1}$, E. Kuznetsova ${ }^{1}$, A. Vinokurov ${ }^{1}$, \\ A. Dunaev', A. Mamoshin ${ }^{1,3}$, I. Snimshchikova ${ }^{1}$, A. Borsukov ${ }^{3}$, A. Bykov ${ }^{4,5,6}$, and I. Meglinski ${ }^{4,5,6,7}$ \\ ${ }^{1}$ Orel State University named after I.S. Turgenev, 95 Komsomolskaya, Orel 302026, Russia \\ ${ }^{2}$ Aston University, Aston Institute of Photonic Technologies, Birmingham, B4 7ET, UK \\ ${ }^{3}$ Smolensk State Medical University, 40 Krupskaya, Smolensk 214019, Russia \\ ${ }^{4}$ Opto-Electronics and Measurement Techniques, Faculty of Information Technology and Electrical \\ Engineering, University of Oulu, Erkki Koiso-Kanttilankatu 3, Oulu 90570, Finland \\ ${ }^{5}$ Interdisciplinary Laboratory of Biophotonics, National Research Tomsk State University, Prospect \\ Lenina 36, Tomsk 634050, Russia \\ ${ }^{6}$ ITMO University, Kronverkskiy Prospect 49, St. Petersburg 197101, Russia \\ ${ }^{7}$ Institute of Biology, Irkutsk State University, 3 Lenin, Irkutsk 664003, Russia \\ E-mail: o.a.stelmashuk@gmail.com
}

\begin{abstract}
The use of liposomal nanoparticles with an incorporated active substance is an innovative and promising approach to diagnostics and therapy. The application of liposomal nanoparticle-based drugs allows for targeted localized delivery, overcomes the natural barriers within the body effectively, and minimizes possible side effects. Liposomes are able to contain a variety of ingredients with practically no limitations to their chemical composition, chemical properties, or size of constituent molecules. This study evaluated the ability to control the passage of fluorescent dye-filled liposomes through the intestinal mucosal barrier after oral administration. For this purpose, the increase in transcutaneous registered fluorescence from tetrabromofluorescein dye was recorded and analysed. Fluorescence intensity was measured at the proximal end of the tail of an animal model after oral administration of the liposomes. Measurements were taken at the excitation wavelengths of 365 and $450 \mathrm{~nm}$. The fluorescence intensity in the group treated with the fluorescent contrast agent encapsulated in liposomal particles increased $140 \%$ of the initial level, but in the group treated with pure contrast agent, the increase in detected fluorescence intensity did not exceed $110 \%$. Mice that received empty liposomes as well as the control group did not demonstrate statistically significant changes in fluorescence intensity. A potential application of our results is an express laser optical method of monitoring the transport of orally administered liposomal particles. The results can be used to help create new optical tools for use in the development of new drugs and in high-throughput screening used during their testing.
\end{abstract}

Keywords: laser fluorescence spectroscopy, liposomes, nanoparticles, drug delivery, optical diagnostics

\section{Introduction}

Currently, non-invasive methods of optical diagnostics based on the principles of spectrophotometry and fluorescence analysis are widely used in medicine, pharmacology, biology, and chemistry. Optical diagnostics and photonics-based techniques are of particular interest in the development of new drugs because of their low cost, versatility, and high sensitivity [1]. The development and testing of a new drug include the study of its metabonomics, which requires a combination of high sensitivity, operability, and ease of automation [2]. Thus, the development of rapid methods of monitoring various biomedical and biotechnological processes is of interest. Optical coherence tomography (OCT) has been used effectively for assessing the efficiency of transcutaneous vaccine delivery [3-5]. In some cases, near-infrared spectroscopy (NIRS) can be used to assess the kinetics of drug release. Furthermore, NIRS and Raman spectroscopy can be used to obtain one spectrum that provides universal and multidimensional information about the drug, including its physical, chemical, and quantitative properties [6]. In addition, optical imaging instruments are being used successfully for the study of drug delivery. Intravital microscopy (IVM) allows non-invasive continuous monitoring of drug movement in the body [7]. Photoacoustic (PA) imaging is used for the simulation of drug transport and the evaluation of the accumulation of substances in an organ or tumour tissue, which is especially important during photodynamic therapy (PDT) [8]. However, these methods have several disadvantages that prevent their widespread use in the biotechnological processes of the pharmaceutical industry. Some disadvantages include difficulty with performing the procedure, the long 
duration of the measurements, the need for a highly skilled technician to run the equipment, and the high cost of equipment. Laser fluorescence spectroscopy (LFS), used in biophotonics, is devoid of many of these disadvantages and can sufficiently automate measurements on a large number of objects of research.

LFS has considerable potential in drug discovery, not only during the development of a new drug formula but also in preclinical and clinical trials. Nanocontainers are increasingly used to deliver a new generation of dosage forms $[9,10]$. One type of nanoscale drug delivery system is the liposome [11], an artificial spherical particle with one or more layers of bimolecular phospholipid [12,13]. The particles have various applications, e.g., they can be used to transport and deliver substances to the target mucosa or skin [14,15]. Use of liposomal nanoparticles is especially attractive because of the possibility of their administration via the oral route, which delivers the content of the nanoparticles directly into the bloodstream [16].

Many drugs are prone to degradation in the environment of the gastrointestinal tract. In addition, the mucosal layer of the small intestine prevents the penetration of many drugs into the bloodstream. High viscosity and mucosal adhesion together with an abundance of auxiliary substances can seriously slow down the spread of the drug through the mucosal layer. This layer is $100-800 \mu \mathrm{m}$ thick and comprises a complex of highly branched glycoproteins, lipids, cellular and serum macromolecules, and other cellular debris [17]. However, the medicament may pass through the gastrointestinal tract with the use of a modified liposome. Studies of intestinal permeability have shown that lipid-based particles can be absorbed if their diameter is not significantly greater than $500 \mathrm{~nm}$ [16]. The transport properties of the liposomal particles can be manipulated by adding a polymer coating to the surface. These coatings can resist degradation in the hostile environment of the gastrointestinal tract, which includes the salts of bile acids and enzymes. For instance, pancreatic lipase typically dissolves the unprotected lipid bilayer [18-20]. A coat of polystyrene polymers or poly(ethylene glycol) (PEG) allows the liposomal particles to penetrate the intestinal mucus [19]. This was, for the first time, confirmed by one research group (Gifu Pharmaceutical University, Japan) that studied the use of orally administered liposomes for targeted delivery of chemotherapeutic agents for intestinal cancer $[20,21]$. This ability to penetrate provides the possibility for unique solutions to certain medical problems, because of the perfect biocompatibility of liposomes as carriers of drugs. In addition, liposomes can contain radioactive, radiopaque, or paramagnetic substances, and substances that reflect ultrasound to improve the quality of images obtained by diagnostic methods such as computed tomography, $\mathrm{X}$-ray, ultrasound, and scintigraphy.

The need to monitor the effectiveness of the penetration of drug carriers must be kept in mind during the development of targeted drug delivery. The penetration of drugs is monitored in preclinical testing by using invasive methods on blood and the tissues of interest in animal models [22,23]. Because these methods are time-consuming and difficult to perform, fluorescence spectroscopy may be an alternative method for assessing the pharmacokinetic processes of liposomal drug penetration. The main purpose of this study was to examine the possibility of using this optical method to control the transport of fluorescent coloured liposomal nanoparticles after oral administration in animal models.

\section{Experimental method and equipment}

A fluorescence channel with a fibre optics probe in series with the multifunctional laser-based non-invasive diagnostic laser system "LAKK-M" (SPE "LAZMA" Ltd, Moscow, Russia) was used to perform fluorescence spectroscopy (Fig. 1) [24,25]. The system provides multiwavelength excitation, detects emission, and processes the fluorescence signal. Its light sources include an LED M365F1 (Thorlabs, Inc., Newton, NJ, USA) (wavelength $=365 \mathrm{~nm}$, power $=1.5 \mathrm{~mW}$ ) and the quantum dot (QD) semiconductor laser LP450-SF15 (Thorlabs) (wavelength $=450 \mathrm{~nm}$, power $=3.5 \mathrm{~mW}$ ), which has high efficiency and low relative intensity noise. The above-mentioned fluorescence excitation powers are provided at the tip of fibre probe, which induces an excitation light flux in the tissue of no more than $0.16 \mathrm{~W} / \mathrm{m}^{2}$ for $365 \mathrm{~nm}$ and $0.37 \mathrm{~W} / \mathrm{m}^{2}$ for $450 \mathrm{~nm}$. The spectrometer was a polychromator with a diffraction grating, and a CCD line (TCD1304AP, Toshiba, Tokyo, Japan) was used as the detector.

The measurement system was previously used to study tissue metabolism by recording the fluorescence of different skin biomarkers [26]. Those studies observed that in probing biological tissue with UV light (e.g., $365 \mathrm{~nm}$ ), the maximum of fluorescence emission of endogenous nicotinamide adenine dinucleotide $(\mathrm{NADH})$ was in the 450-470-nm wavelength range [27]. When excited with blue light (e.g., 430-450 nm), 
the fluorescence peak of flavins was in the range of about 510-520 nm [28]. Thus, the probing depth of this system is sufficient for transcutaneous measurements in animal models.

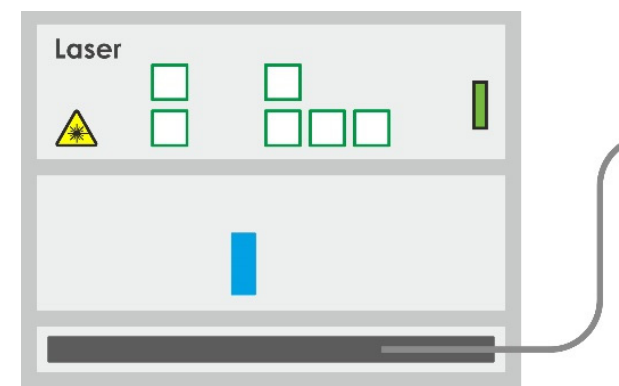

(a)

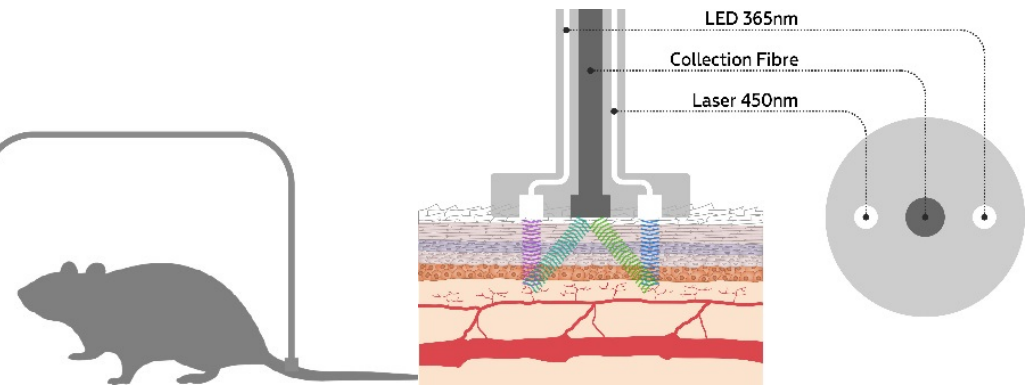

(b)

Figure 1. Schematic representation of the setup used to study the transport of fluorescent coloured liposomal nanoparticles in mice using the "LAKK-M" laser system: a) overall view of the experimental setup; b) fibre optic probe.

The experimental studies complied with EU Directive 2010/63/EU, which defines a humane attitude towards animals and refers to the Three Rs principles (replacement, reduction, and refinement). All studies were approved by the Ethical Committee of Orel State University. The model animals were clinically healthy mice of outbred stock CD-1 that were divided into four groups using the analogue method. Outside of the experiment, the animals were fed the same food. The first group $(n=5)$ was the control group and did not receive any drugs during the experiment. The second group $(n=5)$ received liposomes without dye, the third group $(n=5)$ received pure dye without liposomes, and the fourth group $(n=5)$ received liposomes with incorporated fluorescent dye. The concentration of eosin-Y in the diet of groups 3 and 4 was $5 \mathrm{mg} / \mathrm{kg}$ body weight.

The fluorescence measurements were made on the proximal end of the tail. The area of skin under study was degreased with $96 \%$ ethanol solution before each measurement. The fibre optic probe for measuring fluorescence was placed normal to the surface of the tail. To reduce photobleaching, the tissue was exposed to optical radiation no more than $2 \mathrm{~s}$ per measurement. Before administration of the drug, the background fluorescence at the two excitation wavelengths of 365 and $450 \mathrm{~nm}$ was measured. Fluorescence spectra at those wavelengths were recorded at the time of administration and every $15 \min$ over $2 \mathrm{~h}$.

Liposomes were prepared using a modified version of the reverse-phase evaporation method [29]. Phospholipids, cholesterol, and PEG were dissolved in chloroform. A rotary evaporator was used to remove the organic solvent under vacuum with the water bath at $40{ }^{\circ} \mathrm{C}$ and the rotor speed at $100 \mathrm{rpm}$. Evaporation was stopped after formation of a thin lipid film, which then underwent ultrasound sonication for hydration. The ultrasound treatment continued until the film was flushed and the liposomes formed were less than 500 $\mathrm{nm}$ in diameter. The fluorescent dye eosin-Y (the disodium salt of 2,4,5,7-tetrabromofluorescein), with a maximum intrinsic fluorescence in the $520 \pm 5$-nm region [30-32], was encapsulated inside the liposomes via passive inclusion during lipid film hydration (Fig. 2). 


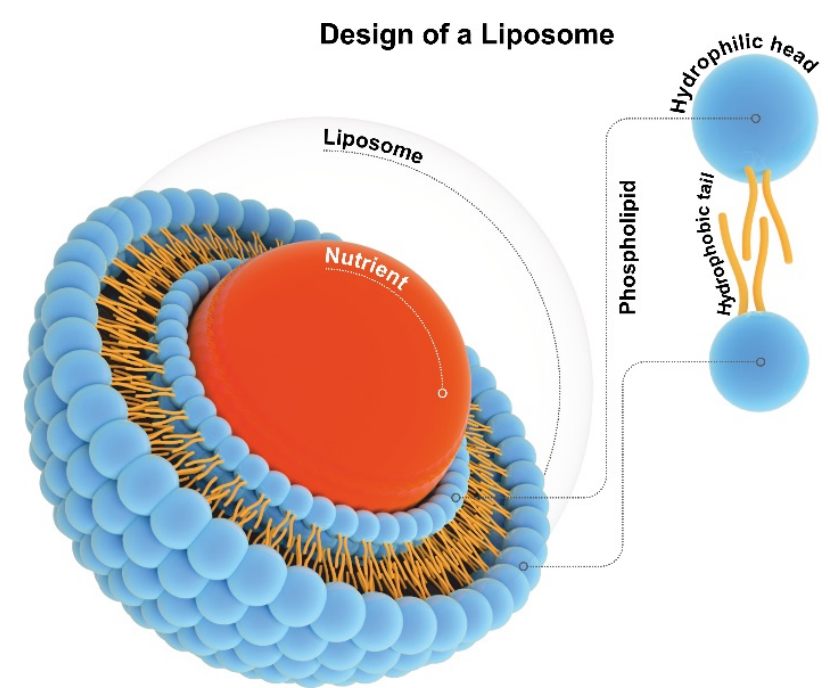

Figure 2. Structure of a liposomal nanoparticle, which was composed of one layer of bimolecular phospholipid that encapsulated the fluorescent dye eosin-Y.

For empty liposomes without the encapsulated dye, the lipid film was hydrated with the sterile saline. Unused eosin-Y was removed from the liposome suspension using dialysis against saline.

\section{Results and discussion}

The main endogenous fluorescent components of mouse skin are collagen and elastin as well as (to a lesser degree) NADH, flavin adenine dinucleotide (FAD), porphyrins, and lipofuscin. Most of these substances fluoresce at 365 and $450 \mathrm{~nm}$, whereas eosin-Y and fluorescein weakly absorb $365 \mathrm{~nm}$ and strongly absorb and fluoresce at $450 \mathrm{~nm}$. Measurements obtained using excitation wavelength $365 \mathrm{~nm}$ did not show a statistically significant difference in fluorescence intensity for all four groups, probably because of the low penetration of the UV excitation signal through the skin where the probe was placed and the low extinction of the dye at that wavelength. The smoothed and averaged spectra for the $450-\mathrm{nm}$ excitation wavelength obtained at the same time (before feeding and 30,60, and $90 \mathrm{~min}$ after feeding) for each group are presented in Figure 3. The spectra show a statistically significant increase in fluorescence intensity in the 480-700$\mathrm{nm}$ wavelength range in the group of mice that received liposomes with encapsulated fluorescent dye. In that group, a pronounced increase (140\% of the initial level) in the average peak fluorescence intensity from $260 \pm 39$ to $420 \pm 36$ a.u. 30 min after liposome administration was observed (Fig. $3 \mathrm{c}$ ). This increase in fluorescence intensity was much greater than that for the group of mice treated with pure dye, i.e., from $283 \pm 42$ to $351 \pm 23$ a.u. (which does not exceed $110 \%$ of the initial level) at the same time points (Fig. 3d). Mice that received empty liposomes and the control group did not demonstrate statistically significant changes in fluorescent intensity (Figs. $3 \mathrm{a}$ and $3 \mathrm{~b}$ ). Table 1 presents the averaged values of the fluorescence spectra maxima for the four groups of mice at seven measurement times.

Table 1. Fluorescence intensity maxima of the four groups of mice

\begin{tabular}{ccccc}
\hline & \multicolumn{4}{c}{ Fluorescence intensity (a.u.) } \\
\cline { 2 - 5 } Time (min) & Control group & $\begin{array}{c}\text { Group received } \\
\text { liposomes }\end{array}$ & $\begin{array}{c}\text { Group received } \\
\text { liposomes with } \\
\text { incorporated } \\
\text { fluorescent dye }\end{array}$ & $\begin{array}{c}\text { Group received } \\
\text { pure fluorescent } \\
\text { dye }\end{array}$ \\
\hline 0 & $259 \pm 53$ & $286 \pm 41$ & $260 \pm 39$ & $283 \pm 42$ \\
15 & $261 \pm 31$ & $286 \pm 53$ & $282 \pm 39$ & $298 \pm 25$ \\
30 & $250 \pm 41$ & $287 \pm 31$ & $420 \pm 36$ & $351 \pm 23$ \\
45 & $251 \pm 19$ & $307 \pm 41$ & $360 \pm 31$ & $333 \pm 39$ \\
60 & $258 \pm 21$ & $290 \pm 19$ & $275 \pm 22$ & $325 \pm 44$ \\
75 & $252 \pm 41$ & $259 \pm 21$ & $290 \pm 42$ & $270 \pm 25$ \\
90 & $249 \pm 41$ & $251 \pm 41$ & $289 \pm 42$ & $260 \pm 41$ \\
\hline
\end{tabular}




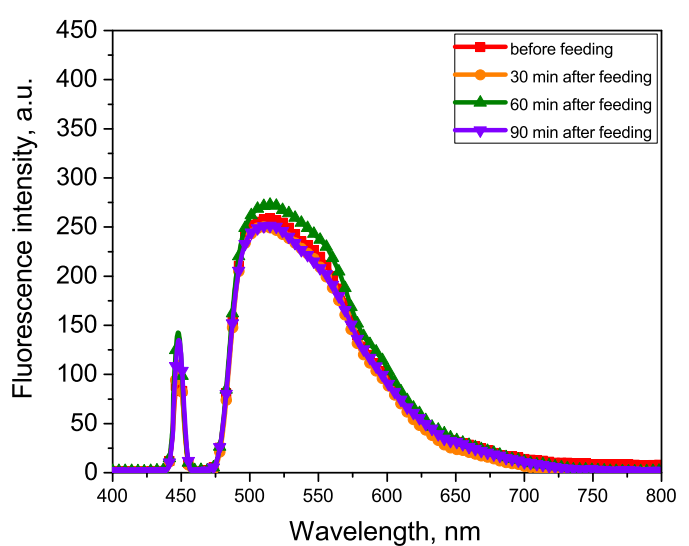

(a)

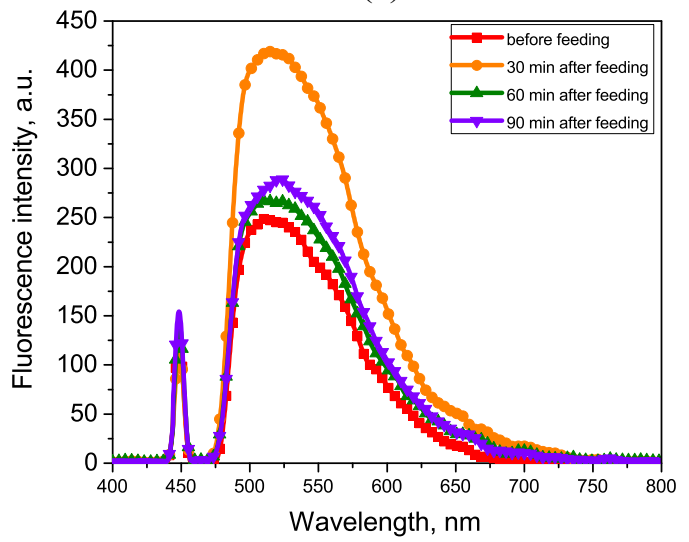

(c)

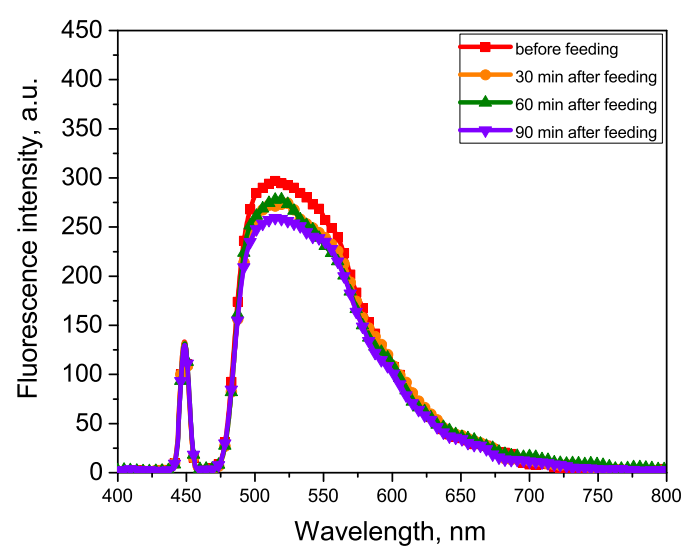

(b)

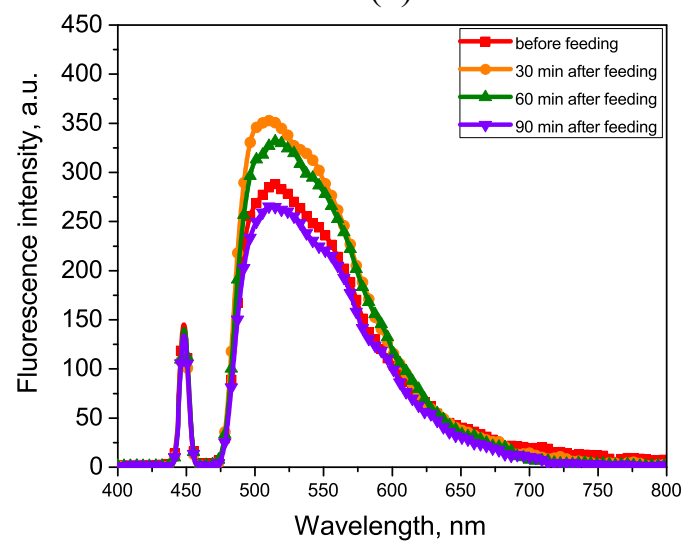

(d)

Figure 3. Fluorescence spectra for 450-nm excitation wavelength (smoothed and averaged for the same time frame for each group): (a) control group; (b) group that received empty liposomes; (c) group that received liposomes with incorporated fluorescent dye; (d) group that received pure fluorescent dye.

The variation in the fluorescence signal as a function of time is presented in Figure 4.

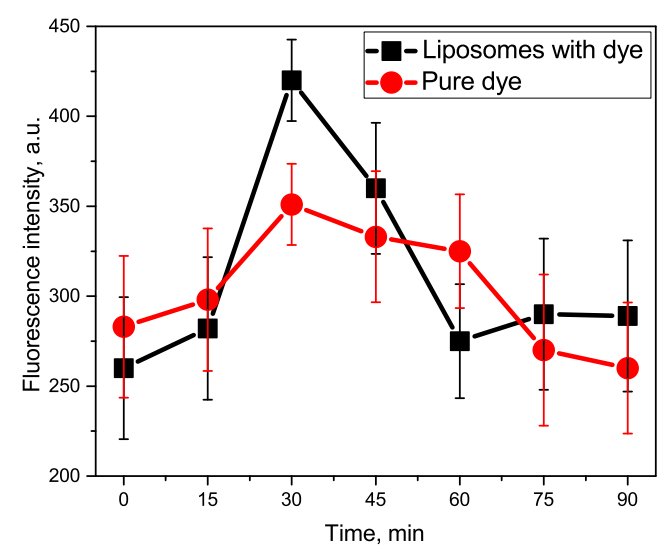

(a)

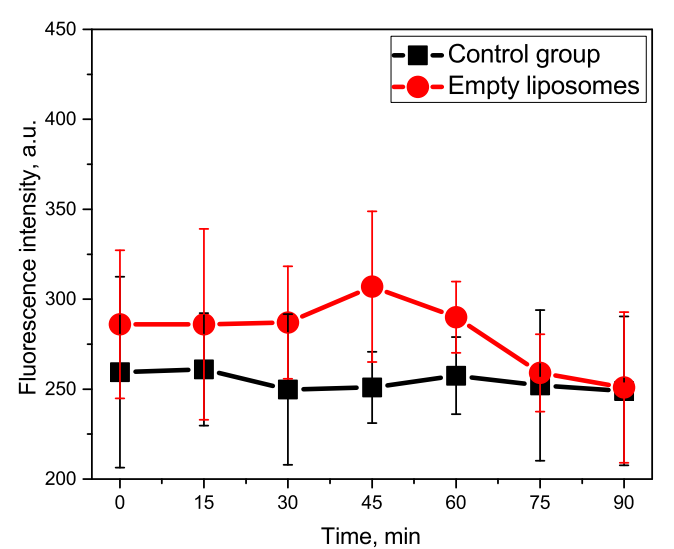

(b)

Figure 4. Fluorescence as a function of time: (a) control group and group that received empty liposomes; (b) group that received liposomes with incorporated fluorescent dye and group that received fluorescent dye.

The PEG coating on the lipid layer allowed the liposomal particles to penetrate the intestinal mucus layer. The results of our experiments show that the multilayer liposomal particles may increase the effectiveness of the transport of the fluorescent dye into the bloodstream via the gastrointestinal tract after oral administration. In addition, some fluorescent dye reached the bloodstream through the intestinal mucosa 
without the assistance of the particles and specifically increased the fluorescence intensity (Figs. $3 \mathrm{~d}$ and 4b).

According to Sercombe et al. [23], PEG greatly affects the pharmacokinetics of liposomes by increasing the circulation time in the blood from 2 to $24 \mathrm{~h}$ in rodents (mice and rats) and more than $45 \mathrm{~h}$ in humans. Circulation time depends of the particle size and the characteristics of the polymer coating. However, this prolongation was not observed in this study. The fluorescence intensity returned to the base level 60-90 min after oral administration.

\section{Conclusion}

We used LFS to investigate the effectiveness of penetration of fluorescently stained liposomes into the circulatory system when administered orally. There was an increase in the fluorescence intensity from $260 \pm 39$ a.u. before administration to $420 \pm 36$ a.u. $30 \mathrm{~min}$ after oral administration of the fluorescent contrast agent encapsulated in liposomal particles. For the group that was administered pure dye, the fluorescence intensity increased slightly from $283 \pm 42$ to $351 \pm 23$ a.u. $30 \mathrm{~min}$ after administration. The possibility of in vivo and in situ express laser optical methods to control the transcutaneous penetration and distribution of liposomal forms designed for oral administration was confirmed. Thus, in addition to the already wide use of LFS, it has the potential for use in a new application. Our results show that it can be used for transcutaneous in vivo measurements and controlled drug administration. Instead of timeconsuming histological analysis of tissue, the LFS approach enables non-invasive measurements and longterm studies on the same animal, which could accelerate the process of drug discovery. The use of LFS can also increase the statistical significance and reliability of clinical trials and reduce the number of animals needed and their suffering. The data obtained by this method can provide information about the pharmacodynamics and the optimal dosage of the drug. The results can then be used for the development of new drugs for transcutaneous delivery and in the high-throughput screening that occurs during testing.

\section{Acknowledgements}

This work was supported by grant No. MK-7168.2016.8 from the President of the Russian Federation for state support of young Russian scientists and by RFBR grant No. 16-32-00662. I Meglinski aknowledges partial support of the Russian Science Foundation (Grant No.15-14-10008). E. Zherebtsov acknowledges for personal support H2020 MSCA-funded project No. 703145.

\section{References}

[1] Cooper M A 2002 Optical biosensors in drug discovery Nat. Rev. Drug Discov. 1 515-28

[2] Rudin M and Weissleder R 2003 Molecular imaging in drug discovery and development Nat. Rev. Drug Discov. 2 123-31

[3] De Beer T, Burggraeve A, Fonteyne M, Saerens L, Remon J P and Vervaet C 2011 Near infrared and Raman spectroscopy for the in-process monitoring of pharmaceutical production processes International Journal of Pharmaceutics 417 32-47

[4] Rattanapak T, Birchall J, Young K, Ishii M, Meglinski I, Rades T and Hook S 2013 Transcutaneous immunization using microneedles and cubosomes: Mechanistic investigations using Optical Coherence Tomography and Two-Photon Microscopy Journal of Controlled Release 172 894-903

[5] Kamali T, Doronin A, Rattanapak T, Hook S and Meglinski I 2012 Assessment of transcutaneous vaccine delivery by optical coherence tomography Laser Physics Letters 9607

[6] Roggo Y, Chalus P, Maurer L, Lema-Martinez C, Edmond A and Jent N 2007 A review of near infrared spectroscopy and chemometrics in pharmaceutical technologies Journal of Pharmaceutical and Biomedical Analysis 44 683-700

[7] Licha $\mathrm{K}$ and Olbrich C 2005 Optical imaging in drug discovery and diagnostic applications Advanced Drug Delivery Reviews 57 1087-108

[8] Tang S, Chen J, Samant P and Xiang L 2016 Photoacoustic image-guided drug delivery in the prostate Proc. SPIE 9709 97090L-6

[9] Petersen A L, Hansen A E, Gabizon A and Andresen T L 2012 Liposome imaging agents in personalized medicine Advanced Drug Delivery Reviews 64 1417-35

[10] Koning G A and Storm G 2003 Targeted drug delivery systems for the intracellular delivery of macromolecular drugs Drug Discovery Today 8 482-3 
[11] Zheng T, Wang J, Wang Q, Nie C, Shi Z, Wang X and Gao Z 2016 A bibliometric analysis of micro/nano-bubble related research: current trends, present application, and future prospects Scientometrics 109 53-71

[12] Vanniasinghe A S, Bender V and Manolios N 2008 The Potential of Liposomal Drug Delivery for the Treatment of Inflammatory Arthritis Seminars in Arthritis and Rheumatism 39 182-96

[13] Zhou X and Zhao G 2015 Global liposome research in the period of 1995-2014: a bibliometric analysis Scientometrics 105 231-48

[14] Jacobi U, Waibler E, Bartoll J, Schulze P, Sterry W and Lademann J 2004 In vivo determination of doxorubicin and its metabolites within the skin using laser scanning microscopy Laser Physics Letters 1 100-3

[15] Lademann O, Richter H, Kramer A, Patzelt A, Meinke M C, Graf C, Gao Q, Korotianskiy E, Ruhl E, Weltmann K D, Lademann J and Koch S 2011 Stimulation of the penetration of particles into the skin by plasma tissue interaction Laser Physics Letters 8 758-64

[16] Vanhoutte G, Verhoye M, Raman E, Roberts M and Linden A V d 2002 In-vivo non-invasive study of the thermoregulatory function of the blood vessels in the rat tail using magnetic resonance angiography NMR in Biomedicine 15 263-9

[17] Lai S K, Wang Y-Y and Hanes J 2009 Mucus-penetrating nanoparticles for drug and gene delivery to mucosal tissues Advanced Drug Delivery Reviews 61 158-71

[18] Koning G A, Kamps J A and Scherphof G L 2002 Efficient intracellular delivery of 5fluorodeoxyuridine into colon cancer cells by targeted immunoliposomes Cancer Detect. Prev. 26 299-307

[19] Karn P R, Vanić Z, Pepić I and Škalko-Basnet N 2011 Mucoadhesive liposomal delivery systems: the choice of coating material Drug Development and Industrial Pharmacy 37 482-8

[20] Mizoue T, Horibe T, Maruyama K, Takizawa T, Iwatsuru M, Kono K, Yanagie H and Moriyasu F 2002 Targetability and intracellular delivery of anti-BCG antibody-modified, pH-sensitive fusogenic immunoliposomes to tumor cells International Journal of Pharmaceutics 237 129-37

[21] Takeuchi H, Matsui Y, Yamamoto H and Kawashima Y 2003 Mucoadhesive properties of carbopol or chitosan-coated liposomes and their effectiveness in the oral administration of calcitonin to rats Journal of Controlled Release 86 235-42

[22] Huang Y Z, Gao J Q, Liang W Q and Nakagawa S 2005 Preparation and characterization of liposomes encapsulating chitosan nanoparticles Biol. Pharm. Bull. 28 387-90

[23] Sercombe L, Veerati T, Moheimani F, Wu S Y, Sood A K and Hua S 2015 Advances and challenges of liposome assisted drug delivery Frontiers in Pharmacology 6 286-13

[24] Dunaev A V, Zherebtsov E A, Rogatkin D A, Stewart N A, Sokolovski S G and Rafailov E U 2013 Substantiation of medical and technical requirements for noninvasive spectrophotometric diagnostic devices J. Biomed. Opt. 18107009

[25] Dremin V V, Zherebtsov E A, Rafailov I E, Vinokurov A Y, Novikova I N, Zherebtsova A I, Litvinova K S, Dunaev A V 2016 The development of attenuation compensation models of fluorescence spectroscopy signals Proc. SPIE 9917 99170Y-6

[26] Dunaev A V, Dremin V D, Zherebtsov E A, Rafailov I E, Litvinova K S, Palmer S G, Stewart N A, Sokolovski S G, Rafailov E U 2015 Individual variability analysis of fluorescence parameters measured in skin with different levels of nutritive blood flow Medical Engineering \& Pphysics 6 574-583

[27] Palmer S, Litvinova K, Dunaev A, Fleming S, McGloin D and Nabi G 2016 Changes in autofluorescence based organoid model of muscle invasive urinary bladder cancer Biomedical Optics Express 7 1193-201

[28] Dremin V V, Sidorov V V, Krupatkin A I, Galstyan G R, Novikova I N, Zherebtsova A I, Zherebtsov E A, Dunaev A V, Abdulvapova Z N, Litvinova K S, Rafailov I E, Sokolovski S G, Rafailov E U 2016 The blood perfusion and NADH/FAD content combined analysis in patients with diabetes foot Proc. SPIE $9698969810-7$

[29] Alves A C, Ramaldes G A, Oliveira M C, Cardoso V N, Mota-Santos T A, Faria A M and Gontijo C M 2008 Ovalbumin encapsulation into liposomes results in distinct degrees of oral immunization in mice Cell Immunol. 254 63-73

[30] Yefimova S L, Kurilchenko I Y, Tkacheva T N, Rozhkov V A, Sorokin A V, Lukianova N Y, Bezdenezhnykh N A, Malyukin Y V and Chekhun V F 2012 Comparative study of dye-loaded liposome accumulation in sensitive and resistant human breast cancer cells Exp. Oncol. 34 101-6

[31] van Rooijen N and van Nieuwmegen R 1978 Fluorochrome staining of multilamellar liposomes Stain. Technol. 53 307-10

[32] Chen C-S, Yao J and Durst R A 2006 Liposome encapsulation of fluorescent nanoparticles: Quantum dots and silica nanoparticles Journal of Nanoparticle Research 8 1033-8 